\title{
Krieg, Frieden und Religion(en) - Hans Jonas' Reflections on Religious Aspects of Warlessness
}

\author{
Raphael Döhn
}

This paper focuses on a posthumously published speech by the Jewish philosopher Hans Jonas, named Reflections on Religious Aspects of Warlessness. Jonas talks about the topic of war and peace in the teachings and history of different religions; namely, Judaism, Christianity, Islam, and Manicheism. Furthermore, he discusses the relations between war, peace, and religion in general, highlights the dangers of the modern nuclear age, and speculates about the role that religion might play in the process of avoiding war in the future. The paper sums up the most important statements of Jonas's speech and takes a look into other texts by Jonas that help us to understand his thoughts on war and peace; e.g., The Concept of God after Auschwitz, his reflections about the participation of Jews in the Second World War and his studies about Gnosticism. In addition, the paper also discusses Jonas's propositions in a questioning manner and takes its own standpoint on both Reflections on Religious Aspects of Warlessness and the relations between war, peace, and the different religions in the past and in the world of today.

Hans Jonas, war, warlessness, peace, violence, Judaism, Christianity, Islam, Manicheism, Gnosticism

Raphael Döhn teaches systematic theology as a research associate at Kassel University. $\mathrm{He}$ is currently working on his doctoral thesis on theodicy, taking a closer look at the works of Hans Jonas, Dorothee Sölle, and Abraham Joshua Heschel. Recent publications: Der werdende Gott als Antwort auf die Theodizeefrage. Perspektiven bei Hans Jonas und in der christlichen Theologie. In: Hutter, Axel / Sans, Georg (Eds.): Zeit Sprache - Gott, Stuttgart (forthcoming 2019). Robert Raphael Geis; Luise Schottroff. In: Roßbach, Nikola (Ed.): Kleines Kasseler Literatur-Lexikon, Hannover 2018, p. 306; 766.

\section{Einleitung}

In diesem Beitrag wird die Rede Reflections on Religious Aspects of Warlessness, die der jüdische Philosoph Hans Jonas am 21. Oktober 1964 im Central Connecticut State College gehalten hat, im Mittelpunkt stehen. ${ }^{1}$ Die lange Zeit unbe-

1 Hans Jonas lebte 1964 im zu Connecticut benachbarten Bundesstaat New York und lehrte dort an der New School for Social Research (vgl. Jonas, Erinnerungen, S. 275-282). Im Folgenden wird in diesem Beitrag, um einen besseren Lesefluss zu ermöglichen, das generische Maskulinum verwendet. 
Raphael Döhn

achtete Rede, 2014 in der kritischen Gesamtausgabe seiner Werke erstmals publiziert, gibt nicht nur Einblicke in Jonas' eigene Überlegungen zu Krieg und Frieden. Sie geht darüber hinaus der Frage nach, wie verschiedene Religionen (Judentum, Christentum, Islam und Manichäismus) über Krieg und Frieden urteilen und hierdurch Einfluss auf historische Verläufe genommen haben und bis in das 20. Jahrhundert hinein nehmen. ${ }^{2}$ Ergänzt werden diese Ausführungen durch Verweise auf weitere Texte, in denen Jonas sich ebenfalls mit diesen Fragen auseinandersetzt, sowie durch Bezugnahmen auf Texte, im Rahmen derer er Aspekte seines Denkens entfaltet, die in enger Beziehung zu seinen Überlegungen zu Krieg und Frieden stehen; z. B. seinen Aufruf zur Beteiligung jüdischer Männer am Zweiten Weltkrieg in dem Text Unsere Teilnahme an diesem Kriege (1939) sowie die Darstellung seines Gottesbildes in der als Theodizeeantwort berühmt gewordenen Rede Der Gottesbegriff nach Auschwitz. Eine jüdische Stimme (1984).

Besondere Bedeutung gewinnt die Auseinandersetzung mit Reflections on Religious Aspects of Warlessness u. a. dadurch, dass Jonas sehr kenntnisreich über verschiedene Religionen und die ihnen inhärenten Kriegs- und Friedenspotenziale spricht. ${ }^{3}$ Im Folgenden werden seine Ausführungen mit Bezug auf religionswissenschaftliche und theologische Literatur in einen größeren Kontext gestellt und kritisch reflektiert. Hierbei wird gezeigt, dass zumindest einige von Jonas' Gedanken auch relevant für die in der Gegenwart stattfindenden Diskurse über Krieg und Frieden im Allgemeinen sowie die Gewalt- und Friedenspotenziale verschiedener Religionen im Speziellen sein können. ${ }^{4}$ Des Weiteren ist Reflections on Religious Aspects of Warlessness weitgehend unberücksichtigt geblieben. ${ }^{5}$ Die durch eine Erörterung des Textes gewonnenen Erkenntnisse

2 Dem in der kritischen Gesamtausgabe abgedruckten Text liegen ein Typoskript (vermutlich eine Mitschrift) sowie ein offensichtlich von Jonas stammender handschriftlicher Stichwortzettel aus Archivbeständen der Universität Konstanz zugrunde (vgl. die editorischen Hinweise zu „Reflections on Religious Aspects of Warlessness“, in: Jonas, Kritische Gesamtausgabe. Bd. III/1, S. 532-533).

3 Es ist jedoch anzumerken, dass das Themenfeld Krieg und Frieden - zumindest explizit - keinen Schwerpunkt in Jonas' Gesamtwerk darstellt, was er auch selbst bei der Verleihung des Friedenspreises des Deutschen Buchhandels im Jahre 1987 betonte (vgl. Jonas, „Technik, Freiheit und Pflicht“, S. 247; vgl. Bongardt, „Der Ökologe und der Krieg“, S. 257-258).

4 Diese aktuellen Diskurse können im Grunde gar nicht wirklich streng voneinander getrennt werden, da in fast allen Diskussionen über Krieg, Frieden und Gewalt vielfältige Verweise auf Religion(en) zu beobachten sind. Vgl. beispielhaft einen Großteil der Beiträge in Werkner / Ebeling, Handbuch Friedensethik.

5 So ließen sich nach intensiver Recherche neben den editorischen Hinweisen und Anmerkungen des Herausgebers in der kritischen Gesamtausgabe nur noch zwei Aufsätze von Michael Bongardt finden, in denen er Jonas' Rede erwähnt (vgl. die editorischen Hinweise und Anmerkungen des Herausgebers zu „Reflections on Religious 
können in Zukunft dafür genutzt werden, andere Texte von Jonas besser zu verstehen und die Forschung zu Jonas' Gesamtwerk voranzubringen. In Ansätzen geschieht dies bereits in diesem Beitrag.

\section{Grundsätzliche Überlegungen}

Interessanterweise unternimmt Jonas in der untersuchten Rede Reflections on Religious Aspects of Warlessness recht ausführliche Versuche, den Begriff Frieden (peace) zu definieren, wohingegen er dies für die Begriffe Krieg (war) sowie Kriegslosigkeit (warlessness) weitgehend unterlässt. ${ }^{6}$ Als erste Beobachtung lässt sich also festhalten, dass sich Jonas dem Thema Krieg, Frieden und Religion(en) über den Friedensbegriff nähert, dessen verschiedene Facetten er beleuchtet. Er hält dabei fest, dass Frieden einen inneren Zustand, aber auch die Beziehung des Menschen zu Gott und die Beziehungen der Menschen untereinander charakterisieren könne. ${ }^{7}$ Diese Mehrdimensionalität - innerer Frieden sowie Frieden mit den Mitmenschen und mit Gott - findet sich in vielen religionswissenschaftlichen oder theologischen Definitionsversuchen des Friedensbegriffes, wobei oftmals Frieden zusätzlich auch auf die Beziehung zwischen Mensch und Natur bezogen wird. ${ }^{8}$

Auffällig ist, dass Jonas die Beziehungen der Menschen untereinander ins Zentrum stellt und diese Beziehungsdimension als wichtigsten Aspekt des Begriffs Frieden ansieht - in einer Rede zu Religionen wäre schließlich auch denkbar gewesen, primär die Beziehung des Menschen zu Gott als seinem Schöpfer in den Blick zu nehmen. Jonas hingegen konzentriert sich auf die Beziehungen zwischen den Menschen, um zu erläutern, dass zumindest aus religiöser Perspektive Frieden weit mehr sei als nur die Abwesenheit von Krieg. Aus diesem Grunde ist bei Jonas keine Polarität von Frieden als etwas immer und nur Gutem sowie Krieg als etwas immer und nur Schlechtem zu beobachten. Da auch ungerechter, unter Tyrannei und Unterdrückung bestehender Frieden möglich sei, könne bisweilen

Aspects of Warlessness“, in: Jonas, Kritische Gesamtausgabe. Bd. III/1, S. 532-533, 636643; vgl. Bongardt, „Der Ökologe und der Krieg“, S. 257-271; vgl. Bongardt, ,„Denn Gott bin ich und nicht ein Mensch““, S. 19).

6 Auch Michael Bongardt übersetzt den Begriff warlessness als Kriegslosigkeit (vgl. Bongardt, „Der Ökologe und der Krieg“, S. 260).

7 Vgl. Jonas, „Reflections on Religious Aspects of Warlessness“, S. 427.

8 Dies zeigt beispielhaft schon ein kurzer Blick in die aktuelle, vierte Auflage der Lexikonreihe Religion in Geschichte und Gegenwart (RGG) und die dort zu findenden Artikel zum Lemma „Frieden“. Die hier genannten vier Dimensionen spielen in allen bzw. fast allen dieser Artikel eine mehr oder weniger bedeutende Rolle - obwohl der Begriff Frieden dort von verschiedenen Autoren aus verschiedenen Perspektiven (u. a. religionswissenschaftlich, dogmatisch und philosophisch) betrachtet wird (vgl. SchmidtLeukel u. a., Art. „Frieden“, Sp. 359-366). 
Raphael Döhn

sogar von einer Pflicht zum Krieg die Rede sein. ${ }^{9}$ Als Beispiel nennt Jonas den Zweiten Weltkrieg: Hier sei es unter ethischen Gesichtspunkten vertretbar gewesen, sich gegen die Bedrohungen, welche ein weiterer globaler Machtzuwachs Hitlers hervorgerufen hätte, zu wehren. Während man nun geneigt sein könnte, zu konstatieren, dass Jonas also zumindest solchen Kriegen, die sinnvoll begründet werden können, positiv gegenüber steht, ist jedoch eine Einschränkung vorzunehmen: Jonas gibt zu bedenken, dass sich die Menschheit mittlerweile nicht nur in der Zeit nach Hitler, sondern zugleich auch in der Zeit nach dem ersten Abwurf einer Atombombe befinde. Durch die Verfügbarkeit von Atomwaffen bestehe nun erstmals die Möglichkeit, dass sich die Menschheit endgültig und vollständig vernichten könnte. Dies führe gewissermaßen zur Bildung einer Allianz von Vertretern aller Religionen sowie von nichtreligiösen Menschen, da an erster Stelle nun das Überleben der Menschheit stehe, das dabei nicht mehr so sehr in Gottes Hand als vielmehr in der Hand der Menschen liege. Jonas fragt daher, ob der Nutzen von Religionen in der Zukunft hauptsächlich darin liegen werde, Menschen zu betäuben und sie so vor dem Eintritt in Kriege zu bewahren. Auch wenn dies bedeuten würde, wie in Aldous Huxleys Roman in einer Brave New World zu leben; also in einer Welt, in der keine wirkliche Freiheit existiert und Menschen die bestehende Weltordnung nicht mehr kritisch hinterfragen. ${ }^{10}$ Ein derartiger Friedenszustand, zu dem dann auch die Religionen beitragen könnten, widerspräche laut Jonas aber der Vorstellung des Menschen als Bild Gottes, da ein Leben unter derartigen Umständen nicht dem entspräche, wie der Mensch sein solle und wie er das Recht habe, zu sein. ${ }^{11}$

Aus einer Perspektive, die Hans Jonas' Gesamtwerk berücksichtigt, können die obigen Ausführungen nicht verwundern, da sie sich recht organisch in Jonas' sonstiges Denken einfügen. Zunächst ist hier die Fokussierung des Friedensbegriffes auf die Beziehungen der Menschen untereinander zu nennen: Diese Konzentration auf das Handeln und die Verantwortung der Menschen zieht sich wie ein roter Faden durch das Jonassche Gesamtwerk und findet nicht nur in seinem ethischen Hauptwerk Das Prinzip Verantwortung (1979), sondern auch in seinem wohl bekanntesten religionsphilosophischen Text - Der Gottesbegriff nach Auschwitz. Eine jüdische Stimme (1984) - ihren Ausdruck. ${ }^{12}$ In dieser als Theodizeeantwort berühmt gewordenen Rede trifft Jonas zwar weitreichende Aussagen über Gott und das Verhältnis von Mensch und Gott; jedoch ist für ihn genauso wichtig, dass durch seine (Haupt-)These, Gott sei nicht allmächtig, die

9 Vgl. Jonas, „Reflections on Religious Aspects of Warlessness“, S. 427-428.

10 Vgl. Jonas, „Reflections on Religious Aspects of Warlessness“, S. 439-444.

11 Vgl. Jonas, „Reflections on Religious Aspects of Warlessness“, S. 428-429, 444.

12 Eine intensivere Auseinandersetzung mit Der Gottesbegriff nach Auschwitz findet im Rahmen meines aktuell in Arbeit befindlichen Dissertationsprojekts statt, das den Arbeitstitel Der Mensch in der Verantwortung. Die Theodizeefrage bei Hans Jonas, Dorothee Sölle und Abraham Joshua Heschel trägt. 
Menschen in die Verantwortung für ihr eigenes Handeln und Nichthandeln genommen werden. Ein Text, der vordergründig primär über Gott spricht, fordert somit die Menschen auf, ihr Handeln zu überdenken und zu ändern. Jonas' Ausführungen zur Theodizeefrage und seine Überlegungen zu Krieg und Frieden weisen hier also gut erkennbar Parallelen auf. ${ }^{13}$ Diese Verknüpfung von Theodizeefrage auf der einen sowie Krieg und Frieden auf der anderen Seite ist durchaus nahe liegend, da sich die Frage stellt, ob und unter welchen Umständen Kriegshandlungen als moralisches Übel zu verstehen sind. ${ }^{14}$ Auch der Verweis auf Huxleys Roman Brave New World taucht durchaus in der theologischen Theodizeeliteratur auf. So weist der christliche Theologe Wilfried Härle - wie Hans Jonas - auf die Problematik eines Lebens wie in der Brave New World hin. Ein derartiges, leidfreies Leben fände in einer Welt statt, die „keine menschlichere Welt [wäre], sondern eine Form der Entmenschlichung, ja eine Art Hölle, weil hier keine menschliche Reifung und kein wirkliches Fühlen mehr möglich wäre. “15 Folgt man den sich ähnelnden kritischen Aussagen von Jonas und Härle zu einem Leben in einer derartigen leid- und kriegsfreien Welt, so müsste man wohl zu dem Schluss kommen, dass eine Welt mit Leid und Kriegen zumindest einer Brave New World vorzuziehen sei. Mit Jonas kann dann noch weitergehend gefragt werden, ob eine solche Brave New World angesichts des Umstandes zu präferieren wäre, dass der nächste (atomare) Weltkrieg das Ende der Menschheit einläuten könnte. Der Blick in den Roman selbst verrät jedoch, dass Huxley hier ein anderes Verhältnis von Religion, Krieg und Leid skizziert. So existiert in der Brave New World keine Religion mehr bzw. es wird bewusst vermieden, Menschen mit Religionen in Berührung kommen zu lassen. Die Bibel ist ein Buch, das der Weltaufsichtsrat Mustafa Mannesmann in seinem Tresor versteckt hält, da Gott als Grund alles Edlen, Erhabenen und Heroischen keinen Platz mehr in der Welt habe. Mannesmann begründet dies folgendermaßen: „Wo es Kriege gibt, Gewissenskonflikte, Versuchungen, denen man widerstehen, und Liebe, die man erkämpfen oder verteidigen muß - dort haben Heldentum und Edelmut selbstverständlich einen gewissen Sinn. Aber heutzutage gibt es keine Kriege mehr."16 Hier wird der Glaube an Gott also keineswegs zur Betäubung der Menschen genutzt, sondern vielmehr gerade deswegen als obsolet betrachtet, weil ein Hinterfragen der Weltordnung sowie der Aufbau (positiver wie negativer)

13 Auch Michael Bongardt deutet Ähnlichkeiten zwischen diesen beiden Texten an, indem er sie innerhalb eines Argumentationszusammenhangs erwähnt (vgl. Bongardt, „,Denn Gott bin ich und nicht ein Mensch““, S. 18-19; vgl. Bongardt, „Der Ökologe und der Krieg“, S. 263-264).

14 Als moralisches Übel (malum morale) wird jenes Übel bezeichnet, das durch menschliche Willensfreiheit verursacht wird (vgl. z. B. von Stosch, Theodizee, S. 7-9).

15 Härle, Dogmatik, S. 460.

16 Huxley, Schöne neue Welt, S. 234. 
Raphael Döhn

emotionaler Beziehungen zu anderen Menschen nicht mehr als sinnvoll angesehen wird. ${ }^{17}$

Die bisher hier angestellten, grundsätzlichen Überlegungen sind nun weiterhin zu beachten, wenn im Folgenden Jonas' Auseinandersetzungen mit den Positionen verschiedener Religionen zum Führen von Kriegen betrachtet werden.

\section{Judentum}

Am ausführlichsten setzt sich Jonas mit dem Judentum auseinander, was nicht verwundert, da der 1903 „im Milieu des liberalen jüdischen Großbürgertums“"18 geborene Jonas sich Zeit seines Lebens als Jude verstanden hat. In den Texten des Tanach findet er vielfältige Beschäftigungen mit Fragen, die Krieg und Frieden betreffen. Er nennt zunächst Josuas Kriege um das Heilige Land: Hier trete Gott noch als eine Art Stammesgott auf, der das Kriegstreiben nicht verurteile, sondern sogar zu Gewalt aufrufe. Aber schon bei den Propheten sei hier eine Verschiebung dahingehend zu erkennen, dass Kriege nun als eine Art göttliche Strafe für Israel interpretiert werden. Diese Entwicklung, bei der andere Völker von Gott hierzu instrumentalisiert werden, gehe einher mit der sich durchsetzenden Vorstellung eines Gottes, der als Herr der Geschichte nicht nur der Gott Israels, sondern aller Völker sei. Durch ein derartiges Verständnis des Gottes Israels als Gott aller Völker werde ein gewichtiger Schritt hin zu einem monotheistischen Glauben gemacht. ${ }^{19}$ Ergänzend zu Jonas' Ausführungen ist daher in Bezug auf das Verhältnis von Religion und Krieg zumindest darauf hinzuweisen, dass bisweilen die These aufgestellt wird, insbesondere monotheistische Religionen - wie Judentum, Christentum und Islam - riefen eher zu Gewalt auf als andere Religionen. Allerdings ist die Validität dieser Aussage umstritten. ${ }^{20}$

17 Vgl. zur Darstellung von Religion in Brave New World auch Schulze, „Imaginative Geschichts-Prophetie bei Huxley und Orwell“, S. 209-217.

18 Wiese, „,Daß man zusammen Philosoph und Jude ist...“", S. 133.

19 Vgl. Jonas, „Reflections on Religious Aspects of Warlessness“, S. 429-431.

20 Vgl. Schieder, „Die Macht der Menschen angesichts der Gewalt des einen Gottes“, S. 53-57; vgl. Bongardt, ,,Denn Gott bin ich und nicht ein Mensch““, S. 7-9. Ähnlich äußert sich Hartmut Zinser: „Als These müßte man zur Diskussion stellen, ob Religionen mit Allgemeingültigkeitsanspruch - vielleicht unerkannt und vielleicht sogar unbeabsichtigt - eine geistige Kriegsführung enthalten und vielleicht sogar betreiben." (Zinser, Religion und Krieg, S. 56). Eine eher kritische Einschätzung findet sich bei Tom Kleffmann: „Die pauschale Redeweise von einer strukturellen Intoleranz des Monotheismus oder einer monotheistischen Sprache der Gewalt ähnelt in ihrer hermeneutischen Dürftigkeit dem Fundamentalismus, den sie bekämpfen will.“ (Kleffmann, „Religionsphilosophische und theologische Überlegungen zur Debatte um Monotheismus und Gewalt“, S. 306). 
Jonas erkennt bei den Propheten den paradoxen Gedanken eines teleologischen Verständnisses der Geschichte: Die Menschheitsgeschichte voller Kriege und Leid steuere auf einen Moment zu, in dem es unter göttlicher Führung zu einem universalen Zustand des Friedens zwischen allen Menschen kommen werde. Jedoch betont er auch, dass laut den Propheten die Aggressoren, die Israel angreifen, den göttlichen Plan nicht kennen, auch keineswegs gerechte Gründe für ihre Kriege haben und daher keinen gerechten Krieg (just war) führen. Die Motive der Aggressoren bleiben demnach vielmehr ungerecht, obschon sie dem Erfüllen des göttlichen Planes dienen und nach dem Willen Gottes handeln. Neben Kriegen dieser Art gebe es aber auch noch eine zweite Art von Krieg, die mit dem Willen Gottes kompatibel sei, wobei hier die handelnden Menschen um die positive Bedeutung ihrer Kriegsführung wissen können. Dies seien Verteidigungskriege, bei denen das Ziel verfolgt werde, das Leben des eigenen Glaubens weiterhin zu ermöglichen. Als Beispiel nennt Jonas hier den Aufstand der Makkabäer: In einem solchen Falle sei der Einsatz von Gewalt legitim, wobei stattdessen auch die Verweigerung von Gewalteinsatz bei gleichzeitiger Verweigerung gegen die feindlichen Mächte möglich sei, was den Märtyrertod zur Folge haben könne. ${ }^{21}$ Hier findet sich also eine Legitimation von Krieg, jedoch mit entscheidenden Einschränkungen: Jonas sieht im Judentum einzig die Möglichkeit gegeben, sich bei einem Verteidigungskrieg auf den göttlichen Willen zu berufen, sofern das Fortbestehen des jüdischen Glaubens gefährdet ist; die aktiv Krieg führenden Aggressoren gegen Israel handeln laut den Propheten zwar auch nach dem Willen Gottes, wissen aber nicht darum. ${ }^{22}$

In Reflections on Religious Aspects of Warlessness beschränkt sich Jonas auf den Tanach und die Zeit des antiken Judentums und klammert daher aus, wie sich die Fragen nach der Rechtmäßigkeit von Kriegen und ihrer Kompatibilität mit dem Gotteswillen weiterhin entwickelt haben und welche Bedeutung diese Prinzipien für die jüdische Lebenspraxis in der Gegenwart haben können. Diese Fragen sind jedoch äußerst relevant, da sich die jüdische Perspektive auf Gewalt im Verlauf des 20. Jahrhunderts durchaus verändert hat. Entscheidende Faktoren hierfür sind die eigene Ohnmacht angesichts der Shoah sowie die Staatsgründung Israels, infolge derer sich die Frage nach dem Umgang mit politischer und mili-

21 Vgl. Jonas, „Reflections on Religious Aspects of Warlessness“, S. 430-433.

22 Vergleicht man die hier von Jonas vorgenommene Darstellung von Gott als Herrn der Geschichte, der innerhalb der Weltgeschichte einen Plan verfolge und unter dessen Führung es zu einem universalen Zustand des Friedens kommen werde, mit seiner in Der Gottesbegriff nach Auschwitz, skizzierten Vorstellung eines nicht-allmächtigen, leidenden und werdenden Gottes, der im Grunde auch keine eschatologische Hoffnung verheißt, so lässt sich durchaus kritisch fragen, inwiefern Jonas' Gottesbegriff mit wichtigen jüdischen Traditionen vereinbar ist. Weiterführend zu dieser Frage vgl. z. B. Goldberg, „Ist Gott allmächtig?“, S. 51-58. 
Raphael Döhn

tärischer Macht auf ganz andere Art und Weise stellt als im Exil. ${ }^{23}$ Weiterhelfen kann hier ein Text von Jonas, der 1939 nach dem Ausbruch des Zweiten Weltkrieges entstanden ist und den Titel Unsere Teilnahme an diesem Kriege trägt. Hier reflektiert er aus einer dezidiert jüdischen Perspektive, ob und wie sich jüdische Männer an diesem Krieg auf Seiten der Alliierten beteiligen sollen. Jonas' Position hierzu ist eindeutig: Ein aktives Mitwirken von Juden an diesem Krieg sei nicht nur vertretbar, sondern vielmehr sogar notwendig, da nur ein Sieg der Alliierten ein Überleben des jüdischen Volkes ermögliche. ${ }^{24}$ Hier lässt sich eine klare Parallele zwischen Reflections on Religious Aspects of Warlessness und Unsere Teilnahme an diesem Kriege erkennen: So wie Jonas 1964 von einer Kompatibilität eines jüdischen Verteidigungskrieges mit dem Gotteswillen spricht, so betont er 1939, dass der gerade beginnende Krieg aus jüdischer Perspektive ohne Berechnung oder Kalkül geführt werden solle, sondern stattdessen als reiner Verteidigungskrieg zur Abwendung der völligen Vernichtung des jüdischen Volkes zu verstehen sei. ${ }^{25}$ Durch die Adressierung an jüdische Männer und Verweise auf das jüdische Volk deutet sich bereits an, dass in diesem Aufruf zur Kriegsteilnahme religiöse Motive eine Rolle spielen. Jonas macht dies durch die von ihm verwendete Terminologie noch deutlicher erkennbar: So sagt er über den Nationalsozialismus, die Juden seien ,sein metaphysischer Feind“26 und spricht von einem ,,bellum judaicum“ “27, da es um das Weiterbestehen des jüdischen Volkes ginge. Aber auch der Fortbestand der jüdischen Ethik, die Einfluss auf die gesamte Menschheit genommen habe, stehe auf dem Spiel. An diesem Punkt kann Jonas daher diesen bellum judaicum in einen größeren Kontext einordnen, indem er diesen Krieg zugleich auch als „bellum christianum“ ${ }^{28}$ bezeichnet, wobei er auf die gemeinsamen Grundlagen christlicher und jüdischer Ethik verweist, die nun im Kontrast zum Nationalsozialismus besonders augenfällig zu Tage treten. Jene jüdisch(-christliche) Ethik, die auch im säkularisierten Europa noch nachwirke, stehe grundsätzlich auf dem Spiel. Der Krieg, der hier von ihm der ,erste Religionskrieg der Moderne“ ${ }^{\text {(29 }}$ genannt wird, verbindet somit dem Jonasschen Gedankengang folgend Juden, Christen und auch Atheisten, da hier zwei grundsätzliche Prinzipien gegeneinander kämpfen: Die von der jüdischen Religion geprägte Zivilisation des modernen Europas auf der einen und der

23 Vgl. Wiese, „Zwischen Ohnmacht und Macht“, S. 93-94.

24 Jonas selbst nahm am Zweiten Weltkrieg teil, wobei er ab 1944 Teil der Jewish Brigade Group war. Hierbei handelte es sich um eine Militäreinheit, die aus jüdischen Soldaten bestand. Zu Jonas' Kriegsteilnahme vgl. Jonas, Erinnerungen, S. 185-214.

25 Vgl. Jonas, „Unsere Teilnahme an diesem Kriege“, S. 74-76.

26 Jonas, „Unsere Teilnahme an diesem Kriege“, S. 62.

27 Jonas, „Unsere Teilnahme an diesem Kriege“, S. 70, 71.

28 Jonas, „Unsere Teilnahme an diesem Kriege“, S. 70-71.

29 Jonas, „Unsere Teilnahme an diesem Kriege“, S. 70. 
Nationalsozialismus auf der anderen Seite. ${ }^{30}$ Laut Christian Wiese ist Jonas dem christlichen Denkern seiner Zeit mit dieser Erkenntnis weit voraus gewesen: „Lange bevor christliche Theologie dies ahnte, beschwor Jonas die zur Bewahrung auch der säkularen ,rational-humanen Zivilisation des modernen Europa notwendige wechselseitige Solidarität von Judentum und Christentum und damit das Paradox, ,daß ein bellum christianum zugleich ein bellum judaicum sein kann'."31

\section{Christentum}

Die Geschichte des Christentums ist Jonas zufolge von einer grundlegenden Ambivalenz geprägt, die bereits in den Evangelien ihren Anfang nehme. ${ }^{32}$ Dort spreche Jesus zwar davon, dass die Sanftmütigen das Erdreich besitzen werden ${ }^{33}$ und man die andere Wange hinhalten solle ${ }^{34}$; dem stehe aber sein Ausspruch gegenüber, er sei nicht gekommen, Frieden zu bringen, sondern das Schwert. ${ }^{35}$ Beispielhaft zeigt sich hier die Pluralität biblischer Aussagen, die es in Bezug auf die Frage nach dem Friedens- und Kriegspotenzial des christlichen Glaubens, aber auch in anderen (theologischen) Fragen so schwer macht, zu eindeutigen Aussagen zu kommen. Dieser Befund gilt ähnlich auch für die heiligen Schriften anderer Religionen, was laut Michael Bongardt zu folgendem Dilemma führt: „Eine der größten Herausforderungen für die Deutung der heiligen Schriften besteht darin, die Pluralität der als geoffenbart geglaubten Aussagen über Gott in eine angemessene Beziehung zueinander zu setzen. ${ }^{“ 36}$ Bongardt kommt daher zu dem Schluss: „[E]s ist ein entscheidender Unterschied, ob Menschen die Gewaltbereitschaft Gottes oder seine Barmherzigkeit für wahr halten. Im letztge-

30 Für eine ausführlichere Auseinandersetzung mit Jonas’ Aufruf vgl. Wiese, ,,Ein Bellum Judaicum in des Wortes tiefster Bedeutung“", S. 92-107 sowie Jonas, Erinnerungen, S. 185-200, 443-444.

31 Wiese, „Ein Bellum Judaicum in des Wortes tiefster Bedeutung““, S. 104.

32 Vgl. Jonas, „Reflections on Religious Aspects of Warlessness“, S. 434.

33 Vgl. Mt 5,5. Dass Jonas hier implizit mehrfach Bezüge zum Matthäusevangelium herstellt, betont auch Michael Bongardt (vgl. Bongardt, „Der Ökologe und der Krieg“, S. 261).

34 Vgl. Mt 5,39.

35 Vgl. Mt 10,34. Ulrich Luz weist in seinem Matthäus-Kommentar darauf hin, dass Jesu Rede vom Schwert hier nicht als Aufruf zu physischer Gewalt zu verstehen sei (vgl. Luz, Das Evangelium nach Matthäus. Teilband 2, S. 133-140). Natürlich schließt das nicht aus, dass dieser Vers in der Geschichte des Christentums trotzdem bisweilen als Aufruf zu Gewalt und Krieg verstanden worden ist.

36 Bongardt, ,,Denn Gott bin ich und nicht ein Mensch““, S. 17. 
Raphael Döhn

nannten Fall wird den Glaubenden ihr Glaube das wichtigste Motiv sein, für eine Befriedung der Religionen einzutreten.“37

Jonas erkennt die bereits in den Evangelien zu beobachtende Ambivalenz auch in der Geschichte des (westlichen) Christentums und merkt an, dass in der Menschheitsgeschichte im Namen keiner anderen Religion so viele Religionskriege geführt worden seien wie im Namen des westlichen Christentums. Besonders zu prüfen ist hierbei Jonas' Begründung für diese Beobachtung: Die Ursache für diese kriegerische Geschichte des Christentums sei, dass im christlichen Glauben die Erlösung der Seele in der Ewigkeit und nicht das physische Wohlergehen in der Zeit im Mittelpunkt stehe. Dementsprechend seien viele Kriege im Namen des Christentums begründet worden, indem darauf verwiesen worden sei, dass die Seelen der Kriegsopfer gerettet werden müssten. Dies erkläre, weshalb die im Namen des christlichen Gottes geführten Kriege oftmals derart grausam und unbarmherzig ausgefochten worden seien. ${ }^{38}$ Diese These kann durchaus auch auf andere Religionen ausgeweitet werden, wobei Jonas das Judentum - im Gegensatz zum Christentum - als eine sehr weltliche Religion sieht, in der Gott als Herr der Geschichte verstanden und die für die Zukunft erhoffte Vollkommenheit als Vollkommenheit dieser Welt (of this world) gedacht werde. ${ }^{39}$ Auch diese Einschätzung des christlichen Glaubens überrascht nicht, da sich derartige Aussagen wiederum in Der Gottesbegriff nach Auschwitz finden lassen. Dort heißt es, die Theodizeefrage sei für Juden bedrängender: „Denn für den Christen, der das wahre Heil vom Jenseits erwartet, ist diese Welt ohnehin weitgehend des Teufels und immer Gegenstand des Mißtrauens, besonders die Menschenwelt wegen der Erbsünde. Aber für den Juden, der im Diesseits den Ort der göttlichen Schöpfung, Gerechtigkeit und Erlösung sieht, ist Gott eminent der Herr der Geschichte ${ }^{\text {40 }}$. Unabhängig davon, ob Jonas’ Beobachtungen im Hinblick auf religiöse Legitimierungsstrategien von Kriegen in der Geschichte des Christentums zutreffen, erscheint seine pauschale Einschätzung des Christentums zu einseitig. So kann der von Jonas dem Christentum unterstellten Diesseitsverachtung durchaus widersprochen werden, da für Christen - wie für Juden -

37 Bongardt, „,Denn Gott bin ich und nicht ein Mensch““, S. 22.

38 Vgl. Jonas, „Reflections on Religious Aspects of Warlessness“, S. 434-436. Als Unterstützung für Jonas' These, nicht das irdische Leben stehe im christlichen Glauben im Mittelpunkt, können die Ausführungen zum Schätzesammeln und Sorgen in Mt 6,1933 herangezogen werden (vgl. die Anmerkungen des Herausgebers zu „Reflections on Religious Aspects of Warlessness“, in: Jonas, Kritische Gesamtausgabe. Bd. III/1, S. 640641).

39 Vgl. Jonas, „Reflections on Religious Aspects of Warlessness“, S. 434.

40 Jonas, „Der Gottesbegriff nach Auschwitz“, S. 409. 
die Welt die gute Schöpfung Gottes darstellt, die darüber hinaus durch Jesus Christus erlöst worden ist. ${ }^{41}$

Abschließend hält Jonas fest, dass anscheinend nicht einmal das Evangelium eine wirkliche Richtschnur für das Leben der Menschen und die grundsätzliche Positionierung zu Kriegen darstellen könne, da in der Geschichte ein und dasselbe Prinzip - die Liebe - zwar immer wieder angeführt worden sei, um die Verbreitung des Christentums auf die gewaltlose Verkündigung des Evangeliums und die Nachfolge Jesu Christi zu beschränken und sich jedweder Gewaltausübung zu verwehren, aber auf der anderen Seite auch das Töten von Menschen mit der Liebe begründet worden sei. ${ }^{42}$ Hiermit spricht Jonas eine grundlegende Herausforderung für die Christen an, die bis heute in der christlichen Theologie noch bearbeitet wird und keineswegs abgeschlossen ist: Wenn der christliche Gott, „dessen Wesen Liebe ist" ${ }^{\text {"43 }}$, die Menschen dazu ruft, aus Liebe zu handeln, was bedeutet das dann konkret? Schließlich kann angesichts der Jonasschen Beobachtungen mit Wilfried Joest und Johannes von Lüpke gefragt werden: „Liebe ist ein großes Wort - aber wenn aus der Liebe in der Praxis je nach Situation und Ermessen alles Beliebige soll folgen können, wird sie da nicht zum bloßen Wort? “44

\section{Islam}

Die dritte Religion, die Jonas untersucht, ist der Islam. Menschen, die Reflections on Religious Aspects of Warlessness aus einer Perspektive des 21. Jahrhunderts und ohne Wissen über die Geschichte des Islams lesen, können hier durchaus verwundert sein. ${ }^{45}$ So gilt der Islam mittlerweile bei vielen Menschen in der westlichen Welt als gewaltsamste aller Religionen, wobei hauptsächlich islamistische Terroranschläge dieses Islambild prägen. Zwar wird diese einseitige Sichtweise oftmals kritisiert - z. B. in theologischer oder religionswissenschaftlicher Fachliteratur -, aber nichtsdestoweniger ist sie in den Medien und v. a. in sozialen Netzwerken sehr präsent. Exemplarisch hält dies Hartmut Zinser fest:

41 Vgl. Schieder, Weltabenteuer Gottes, S. 146-147; vgl. Fischer, Die philosophische Frage nach Gott, S. 123.

42 Vgl. Jonas, „Reflections on Religious Aspects of Warlessness“, S. 436.

43 Härle, Dogmatik, S. 449.

44 Joest / von Lüpke, Dogmatik. Bd. 2, S. 161-162. Joest und von Lüpke nennen hier die Orientierung an Jesus Christus und an biblischen Geboten als mögliche Instrumente, dieser Entleerung des Liebesbegriffes entgegenzuwirken, wobei an Jesus Christus erkannt werde, was Liebe ist, sowie das Liebesgebot und die übrigen Gebote aufeinander bezogen werden können.

45 Vgl. Bongardt, „Der Ökologe und der Krieg“, S. 262. 
Raphael Döhn

„Der Islam gilt im Westen vielfach als eine bellizistische Religion. “46 Ein Blick in den Koran verrate jedoch, dass die Beschäftigung mit Krieg hier nur in einigen Versen eine Rolle spiele und eher andere Themen im Vordergrund stehen. ${ }^{47}$ Zinser ergänzt, dass sich auch im Koran Verse finden lassen, die für ein friedliches Nebeneinander verschiedener Religionen plädieren. Er kommt daher in Bezug auf Krieg und Frieden zu dem Schluss: „Auch der Islam ist wie alle anderen Religionen doppeldeutig. “48 $̈$ Ähnlich äußert sich auch Mariella Ourghi, die im Hinblick auf die islamischen Basistexte (Koran und Hadithe) betont, man könne sowohl einen Zusammenhang von Islam und Friedfertigkeit wie einen Zusammenhang von Islam und Gewalt herstellen. ${ }^{49}$ Jonas hingegen bezeichnet den Islam unter den bisher betrachteten Religionen als diejenige, deren Anhänger sich in der Weltgeschichte am friedfertigsten sowie am wenigsten grausam gezeigt haben und am seltensten an Kriegen beteiligt gewesen seien. Jonas selbst bringt seine Verwunderung darüber zum Ausdruck, dass sich die Muslime in der Geschichte derart friedliebend gezeigt haben, obschon das Führen Heiliger Kriege (holy wars) zu den Grundsätzen des Islams gehöre. ${ }^{50}$ Teilweise ist ein solcher Befund zu erklären, indem - wie im Grunde bei allen Religionen notwendig - bei der Frage nach dem Verhältnis von Krieg und Islam zwei verschiedene Ebenen bedacht und unterschieden werden: Zum einen die Ebene der in normativen Texten gegründeten Lehre und zum anderen die Ebene der heutigen Auslegungen, welche diese Texte auf die gegenwärtige Situation beziehen. ${ }^{51}$

Jonas nennt mehrere Gründe für diese Unterschiede zwischen Lehre und Lebenspraxis: Zunächst den Umstand, dass es nicht zu den Prinzipien des Islams gehöre, andere Menschen unter allen Umständen zur Übernahme des islamischen Glaubens zu zwingen. Sofern die Muslime in einer Region die Übermacht haben, so sei es den religiösen Minderheiten weiterhin erlaubt, ihre Religion auszuüben. Hierin liege der Grund dafür, dass Juden, Christen und andere religiöse Minderheiten in der Islamischen Welt in Kirchen, Synagogen oder Tempeln ihre Religion praktizieren dürfen. Einzig auf der Ebene der politischen Rechte seien die Angehörigen religiöser Minderheiten eingeschränkt. ${ }^{52}$ Außerdem sei unter

46 Zinser, Religion und Krieg, S. 114.

47 Zinser nennt hier als zentrale Themen: „Aussagen über die Einzigartigkeit Gottes, das Paradies, die Hölle und das Jüngste Gericht, die Vorläufer von Mohammed [...]; ferner die Pflichten der Gläubigen, Bekenntnis, Almosen, Gebet" (Zinser, Religion und Krieg, S. 114).

48 Zinser, Religion und Krieg, S. 114.

49 Vgl. Ourghi, „Legitimation von Gewalt im Islam“, S. 104.

50 Vgl. Jonas, „Reflections on Religious Aspects of Warlessness“, S. 436-437.

51 Vgl. Malik, „Gewalt und Gewaltverzicht im Islam“, S. 81-82.

52 Vgl. Jonas, „Reflections on Religious Aspects of Warlessness“, S. 437. Jonas bezieht sich hier auf den so genannten Dhimmi-Status, der Mitgliedern anderer monotheistischer Religionen in von Muslimen regierten Ländern eine begrenzte Freiheit zur Religions- 
Muslimen das Bewusstsein für eine Art Verwandtschaft zwischen Judentum, Christentum und Islam stark verbreitet, was zu der Überzeugung führe, den Anhängern dieser verwandten Religionen müsse mit Toleranz begegnet werden. Darüber hinaus gehe auf Mohammed der Gedanke zurück, bei Juden und Christen handle es sich um die Leute des Buches (people of the book), deren heilige Schriften als Offenbarung anzuerkennen seien. ${ }^{53}$ Das Bewusstsein für eine derartige Verwandtschaft der abrahamitischen Weltreligionen Judentum, Christentum und Islam, das zu gegenseitiger Toleranz führen soll, wird oftmals auch von jüdischen und christlichen Denkern betont. So hält der jüdische Theologe Abraham Joshua Heschel fest: „Ein Jude sollte ernsthaft über die Verantwortung nachdenken, die sich aus der jüdischen Geschichte ergibt, nämlich, die Mutter zweier Weltreligionen zu sein. “54

\section{Manichäismus}

An die Auseinandersetzung mit den drei abrahamitischen Weltreligionen, die auch heute noch eine gewichtige Rolle in der Welt spielen, schließt sich in Reflections on Religious Aspects of Warlessness die Beschäftigung mit einer vierten, mittlerweile gewissermaßen ausgestorbenen Religion an: Dem Manichäismus. ${ }^{55}$ Jonas wählt diese Religion aus, da nur bei ihr der Aufruf zur Gewaltlosigkeit bereits in den Grundlagen der Religion verankert sei. Nach manichäischer Überzeugung sei die göttliche Substanz im Schöpfungsvorgang zerstreut worden, weswegen sich in allen Formen des Lebens etwas Göttliches finden lasse. Aus diesem Grunde solle niemand anderen Menschen bzw. anderen empfindungsfähigen Kreaturen Schmerz zufügen. Da ein solches Zufügen von Schmerz Sünde sei, solle das Verletzen anderer Kreaturen so gut wie möglich vermieden werden. In ihrer Konsequenz führe diese Auffassung dann nicht nur zu einem radikalen Pazifismus im Namen der Religion, sondern auch zu einem sehr asketischen und quietistischen Leben. Schließlich könne man sogar so weit gehen, zu sagen, dass jeder, der den Boden bearbeite oder sich auch nur durch die Luft bewege, den

ausübung erlaubt, die an Auflagen gebunden ist. Diese auf der Basis von Sure 9:7 und 9:10 entwickelten Regelungen werden und wurden historisch wie regional unterschiedlich gehandhabt (vgl. die Anmerkungen des Herausgebers zu „Reflections on Religious Aspects of Warlessness“, in: Jonas, Kritische Gesamtausgabe. Bd. III/1, S. 642).

53 Vgl. Jonas, „Reflections on Religious Aspects of Warlessness“, S. 437-438. Jonas referiert hier auf Sure 2:62, in der auch die Sabäer als weitere Gruppe genannt werden. Ob dieses Prinzip auf weitere Religionen auszuweiten sei, wird unter Muslimen diskutiert (vgl. die Anmerkungen des Herausgebers zu „Reflections on Religious Aspects of Warlessness“, in: Jonas, Kritische Gesamtausgabe. Bd. III/1, S. 642).

54 Heschel, „Keine Religion ist ein Eiland“, S. 332.

55 Wesentlich ausführlicher setzt sich Hans Jonas mit dem Manichäismus auseinander in Jonas, Gnosis und spätantiker Geist. Erster Teil, S. 284-320. 
Raphael Döhn

Boden oder die Luft verletze und somit zu dem Leiden Gottes beitrage. Da sich unter Beachtung dieser Maxime aber kaum leben lasse, gelten diese Vorgaben in ihrer extremen Ausprägung nur für eine Gruppe Erwählter (elect), wohingegen die übrigen Gläubigen diese Vorgaben nicht einhalten müssen. ${ }^{56}$

Tatsächlich ist Jonas darin Recht zu geben, dass die Auseinandersetzung mit dem Manichäismus interessante Erkenntnisse in Bezug auf das Thema Krieg, Frieden und Religion(en) liefern kann. So ist zunächst festzuhalten, dass das Grundprinzip des Nichtverletzens anderer Kreaturen jedwede Teilnahme an Kriegshandlungen sowie das Töten von Menschen im Grunde unmöglich macht. ${ }^{57}$ Obschon ein prinzipielles Tötungsverbot auch in anderen Religionen bekannt ist, gilt der Manichäismus in der Forschungsliteratur trotzdem ,als radikalster Versuch der Gewaltlosigkeit" ${ }^{\text {"58 }}$, wobei besonders eindrücklich die oben bereits angesprochene Annahme einer Empfindungsfähigkeit von Erde, Pflanzen, Wasser und Luft ist, was Aktivitäten wie Ackerbau, Ernten, Essen, Waschen oder sogar Gehen bereits als Gewalttaten erscheinen lässt. ${ }^{59}$

Jedoch drängen sich hier weitere Überlegungen auf: Zunächst ist die Frage zu stellen, ob die Vorstellung einer derartigen Empfindungsfähigkeit von Erde, Pflanzen, Wasser und Luft sowie die daraus folgenden Konsequenzen eigentlich als lebensdienlich bzw. lebenstauglich angesehen werden können oder nicht. Jonas selbst spricht dies in seinem zentralen Werk Gnosis und spätantiker Geist an; dort heißt es im Hinblick auf das manichäische Prinzip des Nichtverletzens: „Doch schon daß diese Haltung der Schonung unterschiedslos auf die ganze Natur, selbst die unbeseelte, und fast mehr auf die Tier- und Pflanzenwelt als die menschliche sich erstreckt, spricht weniger für ein konkret ethisches als ein abstrakt dogmatisches Prinzip, das mit dem Sein der Person wenig zu tun hat." ${ }^{60}$ Aber auch auf die Motivation, die hier zur Gewaltlosigkeit führt, muss hingewiesen werden. Jonas deutet in Reflections on Religious Aspects of Warlessness an,

56 Vgl. Jonas, „Reflections on Religious Aspects of Warlessness“, S. 438-439. Ergänzend zum Manichäismus erwähnt Jonas hier noch knapp den Buddhismus. Grundsätzlich sind in Bezug auf den Umgang mit Gewalt durchaus Ähnlichkeiten zwischen Manichäismus und Buddhismus zu erkennen. Dies lässt sich zum Teil dadurch erklären, dass der Gründer dieser Religion - Mani - wohl von der buddhistischen Lehre wusste (vgl. Burkert, „Zum Umgang der Religionen mit Gewalt“, S. 195). Der Manichäismus wird oftmals als synkretistische Religion bzw. als Ergebnis einer Religionssynthese verstanden, in die u.a. Elemente des Judentums, des Christentums und des Buddhismus eingeflossen seien (vgl. van Oort, Art. „Manichäismus“, Sp. 740; vgl. Markschies, Die Gnosis, S. 101102).

57 Vgl. Burkert, „Zum Umgang der Religionen mit Gewalt“, S. 196; vgl. van Oort, Art. „Manichäismus“, Sp. 739.

58 Burkert, „Zum Umgang der Religionen mit Gewalt“, S. 189.

59 Vgl. Burkert, „Zum Umgang der Religionen mit Gewalt“, S. 192-196.

60 Jonas, Gnosis und spätantiker Geist. Zweiter Teil, S. 37. 
dass die Schöpfung nach manichäischem Glauben nicht gottgewollt sei und die Zerstreuung der göttlichen Substanz in der Welt eine göttliche Tragödie (divine tragedy) darstelle. ${ }^{61}$ Auch hier hilft ein Blick in Gnosis und spätantiker Geist weiter, wo Jonas in seiner Darstellung des manichäischen Schöpfungsmythos und der aus ihm folgenden Ethik aufzeigt, dass die Existenz der Pflanzen, Tiere und Menschen hier nicht als etwas Positives und Wertzuschätzendes angesehen werde, sondern vielmehr als etwas zu Überwindendes, wobei das Prinzip der Gewaltlosigkeit dazu diene, die in der Welt gefangene göttliche Substanz nicht zu verletzen und sie nicht in der (bösen) Welt zu binden. ${ }^{62}$ Die als Ziel verfolgte Gewaltlosigkeit resultiert hier also gerade nicht aus einer Wertschätzung für die Schöpfung und die in ihr lebenden Geschöpfe, sondern aus einer gegenteiligen Sichtweise. Hierauf die eigene Ethik fußen zu lassen, erscheint insbesondere aus den Perspektiven der drei abrahamitischen Weltreligionen, in denen an Gott als gütiger Schöpfer der Welt und der sie bevölkernden Lebewesen geglaubt wird, nicht sinnvoll.

Trotz der kritischen Perspektive, die man somit durchaus auf den Manichäismus einnehmen kann, wäre im Anschluss an Walter Burkert ebenfalls zu bedenken, ob es ein Zufall ist, dass unter jenen Religionen, die Hans Jonas in Reflections on Religious Aspects of Warlessness behandelt, ausgerechnet der Manichäismus heute - anders als Judentum, Christentum und Islam - keine tragende Rolle mehr in der Weltgeschichte spielt. Kann eine Religion, die in Lehre und Lebenspraxis derart pazifistisch eingestellt ist und auf Gewaltlosigkeit pocht, vielleicht in unserer Welt überhaupt nicht überdauern? Und wenn ja, was sagt dies über unsere Welt aus ? ${ }^{63}$ Oder in Burkerts Worten: „Ist nach dem Urteil der Weltgeschichte die gewaltlose Religion eine von vornherein zum Untergang verurteilte Option? [Judentum, Anm. R.D.] Christentum, Islam und Buddhismus haben sich je in ihrer Weise als welttauglicher erwiesen." ${ }^{64}$

\section{Schlussbemerkungen}

Es hat sich gezeigt, dass eine Beschäftigung mit Reflections on Religious Aspects of Warlessness erweiterte Perspektiven auf das bereits intensiv erforschte Gesamtwerk von Hans Jonas bzw. auf andere seiner Texte zu eröffnen vermag. Beispiele hierfür innerhalb dieses Beitrags sind die Verknüpfungen, die zu Unsere Teilnahme an diesem Kriege und Der Gottesbegriff nach Auschwitz hergestellt

61 Vgl. Jonas, „Reflections on Religious Aspects of Warlessness“, S. 438.

62 Vgl. Jonas, Gnosis und spätantiker Geist. Erster Teil, S. 307-310, 313-315.

63 Natürlich soll hier nicht der Eindruck entstehen, dass der Aufruf zur Gewaltlosigkeit historisch als der einzige oder zwingende Grund für den Niedergang des Manichäismus zu verstehen sei. Dies wäre sicherlich zu einfach gedacht.

64 Burkert, „Zum Umgang der Religionen mit Gewalt“, S. 199. 
Raphael Döhn

wurden sowie die Interpretation seiner Ausführungen zum Islam, da er sich mit dieser Religion weit weniger oft und ausgiebig in anderen Schriften auseinandergesetzt hat als mit Judentum, Christentum und Manichäismus.

Darüber hinaus erscheint es aber auch lohnenswert, Reflections on Religious Aspects of Warlessness zukünftig in den aktuellen Diskursen über Krieg, Frieden und die Gewalt- und Friedenspotenziale von Religionen zur Sprache zu bringen. Ein Ansatz könnte hierbei sein, Jonas' Beobachtungen zu Gemeinsamkeiten und Unterschieden zwischen den verschiedenen Religionen (insbesondere von Judentum, Christentum und Islam) kritisch zu prüfen und hieraus z. B. Rückschlüsse für den interreligiösen Dialog zu gewinnen. Zum anderen wäre es auch möglich, Jonas' Rede primär als ein Stück Zeitgeschichte zu betrachten und zu fragen, ob und wie sich der Blick auf das Verhältnis von Krieg, Frieden und Religion(en) in den vergangenen Jahrzehnten gewandelt hat.

\section{Literaturverzeichnis}

Bongardt, Michael: „Der Ökologe und der Krieg“, in: Nielsen-Sikora, Jürgen / Gordon, John-Stewart (Hrsg.): Hans Jonas. Zur Diskussion seiner Denkwege (Hans Jonas. Volume 1), Berlin 2017, S. 257-271.

Burkert, Walter: „Zum Umgang der Religionen mit Gewalt: Das Experiment des Manichäismus“, in: Berliner Theologische Zeitschrift 1/13 (1996), S. 184-199.

Fischer, Norbert: Die philosophische Frage nach Gott. Ein Gang durch ihre Stationen (AMATECA. Bd. 2), Paderborn 1995.

Goldberg, Arnold: „Ist Gott allmächtig? Was die Rabbinen Hans Jonas antworten könnten“, in: Judaica 1-2/47 (1991), S. 51-58.

Härle, Wilfried: Dogmatik, 4. Aufl. Berlin u. a. 2012.

Heschel, Abraham Joshua: „Keine Religion ist ein Eiland“, in: Rothschild, Fritz A. (Hrsg.): Christentum aus jüdischer Sicht. Fünf jüdische Denker des 20. Jahrhunderts über das Christentum und sein Verhältnis zum Judentum. Übersetzt von Rudnick, Ursula / Olmesdahl, Ruth, Berlin u. a. 1998, S. 324-341.

Huxley, Aldous: Schöne neue Welt. Ein Roman der Zukunft. Übersetzt von Herlitschka, Herberth E., 64. Aufl. Frankfurt am Main 2007.

Joest, Wilfried / Johannes von Lüpke: Dogmatik. Bd. II: Der Weg Gottes mit dem Menschen, 5. Aufl. Göttingen 2012.

Jonas, Hans: „Technik, Freiheit und Pflicht. Dankesrede anläßlich der Verleihung des Friedenspreises des Deutschen Buchhandels am 11. Oktober 1987 in Frankfurt am Main“, in: Jonas, Hans: Kritische Gesamtausgabe. Bd. I/2: Das Prinzip Verantwortung. Zweiter Teilband: Tragweite und Aktualität einer Zukunftsethik. Herausgegeben von Böhler, Dietrich / Herrmann, Bernadette, Freiburg im Breisgau u.a. 2017, S. 247-256.

Jonas, Hans: „Das Prinzip Verantwortung. Versuch einer Ethik für die technologische Zivilisation“, in: Jonas, Hans: Kritische Gesamtausgabe. Bd. I/2: Das Prinzip Verantwortung. Erster Teilband: Grundlegung. Herausgegeben von Böhler, Dietrich / Herrmann, Bernadette, Freiburg im Breisgau u. a. 2015, S. 1-420. 
Jonas, Hans: „Der Gottesbegriff nach Auschwitz“, in: Jonas, Hans: Kritische Gesamtausgabe. Bd. III/1: Metaphysische und religionsphilosophische Studien. Herausgegeben von Bongardt, Michael / Lenzig, Udo / Müller, Wolfgang Erich, Freiburg im Breisgau u. a. 2014, S. 407-426.

Jonas, Hans: „Reflections on Religious Aspects of Warlessness“, in: Jonas, Hans: Kritische Gesamtausgabe. Bd. III/1: Metaphysische und religionsphilosophische Studien. Herausgegeben von Bongardt, Michael / Lenzig, Udo / Müller, Wolfgang Erich, Freiburg im Breisgau u.a. 2014, S. 427-444. [zusätzliche editorische Hinweise und Anmerkungen des Herausgebers: S. 532-533, 636-643].

Jonas, Hans: „Unsere Teilnahme an diesem Kriege“, in: Jonas, Hans: Kritische Gesamtausgabe. Bd. III/2: Herausforderungen und Profile. Jüdisch-deutscher Geist in der Zeit-gegen die Zeit. Herausgegeben von Lalla, Sebastian / Preußger, Florian / Böhler, Dietrich, Freiburg im Breisgau u. a. 2013, S. 61-76.

Jonas, Hans: Erinnerungen. Nach Gesprächen mit Rachel Salamander. Herausgegeben von Wiese, Christian, Frankfurt am Main u. a. 2003.

Jonas, Hans: Gnosis und spätantiker Geist. Zweiter Teil: Von der Mythologie zur mystischen Philosophie. Erste und zweite Hälfte. Herausgegeben von Rudolph, Kurt (Forschungen zur Religion und Literatur des Alten und Neuen Testaments. Bd. 159), Göttingen 1993.

Jonas, Hans: Gnosis und spätantiker Geist. Erster Teil: Die mythologische Gnosis. Mit einer Einleitung zur Geschichte und Methodologie der Forschung (Forschungen zur Religion und Literatur des Alten und Neuen Testaments. Bd. 33), 4. Aufl. Göttingen 1988.

Kleffmann, Tom: „Religionsphilosophische und theologische Überlegungen zur Debatte um Monotheismus und Gewalt", in: Leuzinger-Bohleber, Marianne / Klumbies, PaulGerhard (Hrsg.): Religion und Fanatismus. Psychoanalytische und theologische Zugänge, Göttingen 2010, S. 294-307.

Luz, Ulrich: Das Evangelium nach Matthäus. Teilband 2: Mt 8-17 (Evangelisch-Katholischer Kommentar zum Neuen Testament. Bd. I/2), 4. Aufl. Düsseldorf u. a. 2007.

Malik, Jamal: „Gewalt und Gewaltverzicht im Islam“, in: Bultmann, Christoph / Kranemann, Benedikt / Rüpke, Jörg (Hrsg.): Religion. Gewalt. Gewaltlosigkeit. Probleme - Positionen - Perspektiven, Münster 2004, S. 70-82.

Markschies, Christoph: Die Gnosis, 3. Aufl. München 2010.

Oort, Johannes van: Art. „Manichäismus“, in: Religion in Geschichte und Gegenwart. Bd. 5, 4. Aufl. Tübingen 2002, Sp. 732-741.

Ourghi, Mariella: „Legitimation von Gewalt im Islam“, in: Lederhilger, Severin J. (Hrsg.): Gewalt im Namen Gottes. Die Verantwortung der Religionen für Krieg und Frieden. 16. Ökumenische Sommerakademie Kremsmünster 2014 (Linzer philosophisch-theologische Beiträge. Bd. 30), Frankfurt am Main 2015, S. 97-109.

Schieder, Rolf: „Die Macht der Menschen angesichts der Gewalt des einen Gottes“, in: Lederhilger, Severin J. (Hrsg.): Gewalt im Namen Gottes. Die Verantwortung der Religionen für Krieg und Frieden. 16. Ökumenische Sommerakademie Kremsmünster 2014 (Linzer philosophisch-theologische Beiträge. Bd. 30), Frankfurt am Main 2015, S. $45-59$. 
Raphael Döhn

Schieder, Thomas: Weltabenteuer Gottes. Die Gottesfrage bei Hans Jonas (Abhandlungen zur Philosophie, Psychologie, Soziologie der Religion und Ökumenik. N. F. Bd. 48) 2. Aufl. Paderborn 1998.

Schmidt-Leukel, Perry u. a.: Art. „Frieden“, in: Religion in Geschichte und Gegenwart. Bd. 3, 4. Aufl. Tübingen 2000, Sp. 359-366.

Stosch, Klaus von: Theodizee, Paderborn 2013.

Werkner, Ines-Jacqueline / Ebeling, Klaus (Hrsg.): Handbuch Friedensethik, Wiesbaden 2017.

Wiese, Christian: „Zwischen Ohnmacht und Macht. Akzente jüdischer Auseinandersetzung mit der Gewaltproblematik im Kontext politischen Handelns im 20. Jahrhundert", in: Bultmann, Christoph / Kranemann, Benedikt / Rüpke, Jörg (Hrsg.): Religion. Gewalt. Gewaltlosigkeit. Probleme - Positionen - Perspektiven, Münster 2004, S. 93-115.

Wiese, Christian: „,Daß man zusammen Philosoph und Jude ist... Z Zur Dimension des Jüdischen in Hans Jonas' philosophischer Ethik der Bewahrung der ,Schöpfung ““, in: Valentin, Joachim / Wendel, Saskia (Hrsg.): Jüdische Traditionen in der Philosophie des 20. Jahrhunderts, Darmstadt 2000, S. 131-147.

Wiese, Christian: „,Ein Bellum Judaicum in des Wortes tiefster Bedeutung“. Hans Jonas’ Kriegsaufruf 1939 im Kontext seiner Biographie und seines philosophischen Denkens“, in: Birkenhauer, Anne (Hrsg.): Jüdischer Almanach des Leo Baeck Instituts 2001/5761, Frankfurt am Main 2000, S. 92-105.

Zinser, Hartmut: Religion und Krieg, Paderborn 2015.

\section{Internetquellen}

Bongardt, Michael: „,Denn Gott bin ich und nicht ein Mensch“ (Hosea 11,9). Zur verhängnisvollen Allianz von Religion und Gewalt", in: Pastoraltheologische Informationen 1/36 (2016), S. 7-22, online abrufbar unter: https://www.uni-muenster.de/ Ejournals/index.php/pthi/article/view/2110 [15.01.2018].

Schulze, Fritz W.: „Imaginative Geschichts-Prophetie bei Huxley und Orwell“, in: Zeitschrift für Religions- und Geistesgeschichte 3/36 (1984), S. 204-222, online abrufbar unter: http://booksandjournals.brillonline.com/content/journals/10.1163/1570073 $84 \times 00023$ [15.01.2018].

Raphael Döhn, Department of Protestant Theology, University of Kassel, Henschelstraße 2, 34127 Kassel, Germany, e-mail: raphael.doehn@uni-kassel.de

Citation: Döhn, Raphael: „Krieg, Frieden und Religion(en) - Hans Jonas’ Reflections on Religious Aspects of Warlessness", in: Kallhoff, Angela / Schulte-Umberg, Thomas (eds.): Moralities of Warfare and Religion (J-RaT 2018 / 1) pp. 189-206.

Datum der Publikation: 16.07.2018 